AperTO - Archivio Istituzionale Open Access dell'Università di Torino

\title{
Mechanical and biological treatment of long bone non-unions
}

\section{This is the author's manuscript}

Original Citation:

Availability:

This version is available http://hdl.handle.net/2318/34995

since

Terms of use:

Open Access

Anyone can freely access the full text of works made available as "Open Access". Works made available under a Creative Commons license can be used according to the terms and conditions of said license. Use of all other works requires consent of the right holder (author or publisher) if not exempted from copyright protection by the applicable law. 


\title{
Mechanical and biological treatment of long bone non-unions
}

\author{
Antonio Biasibetti ${ }^{a, *}$, Domenico Aloj ${ }^{a}$, Giuseppe Di Gregorio ${ }^{a}$, \\ Alessandro Massè ${ }^{\mathrm{b}}$, Carlo Salomone
}

\author{
a Unità Operativa di Traumatologia Muscolo Scheletrica e Fissazione Esterna, \\ Centro Traumatologico Ortopedico, Via Zuretti n. 29, 10126 Torino, Italy \\ b I Clinica Ortopedica dell'Università degli studi di Torino, Centro Traumatologico Ortopedico, \\ Via Zuretti n. 29, 10126 Torino, Italy
}

\section{KEYWORDS \\ External fixation; \\ Non-union; \\ llizarov; \\ BMPs; \\ Bone grafting}

Summary Non-union (NU) of bones is a multifactorial phenomenon. Infected nonunions and/or those with bone loss require "biological treatment", such as the excision of the pathological infected non-union site or the "creation" of new bone substance by the use of distraction techniques.

The treatment of choice that meets both the biological and mechanical requirements is that of the external fixation, in as much as it has an extremely low septic complication rate and the mechanical environment can be varied automatically or programmed depending on the requirements.

The authors report on a total of 287 cases of non-union treated with external fixation, with a $93 \%$ success rate.

(C) 2005 Elsevier Ltd. All rights reserved.

\section{Introduction}

To date, there is no universal accepted standardization of definition of non-union of fractures.

Various criteria may be used to arrive at a definition, depending on how the healing process is defined. The term "non-union" could be used to describe fracture that requires a more than average time lapse to arrive at a successful outcome.

Classification of non-union is of paramount importance allowing development of treatment

\footnotetext{
* Corresponding author. Tel.: +39 11 6933578/63665

E-mail address: abiasib@tin.it (A. Biasibetti).
}

strategies but also communication amongst clinicians. Classification should be based on two principles: knowledge of the biological situation and the treatment indication. In our unit, classification of non-union is based on radiographic evaluation.

Type 1 non-unions are hypertrophic with an elephant's foot-like shape or a horizontal rim or stabilizing shelf. In this case, the mechanical stabilization is achieved by compression, ${ }^{7}$ whatever the chosen form (Fig. 1).

Type 2 non-unions are a more complex type of fracture, oblique with large fragments, in which a purely axial compression would generate torsional or cutting forces, with a consequential negative 
(a)

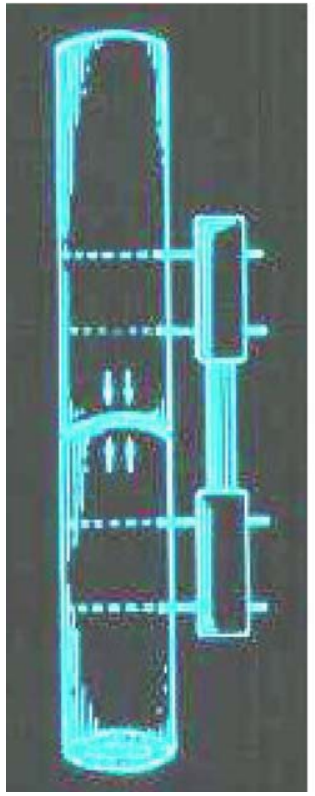

(b)

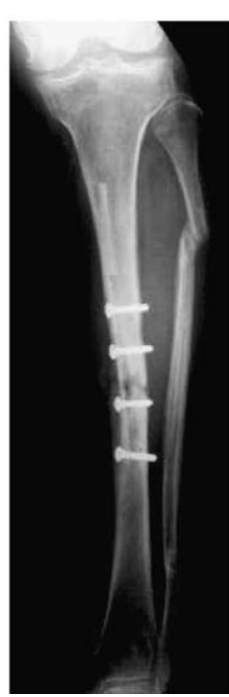

(c)

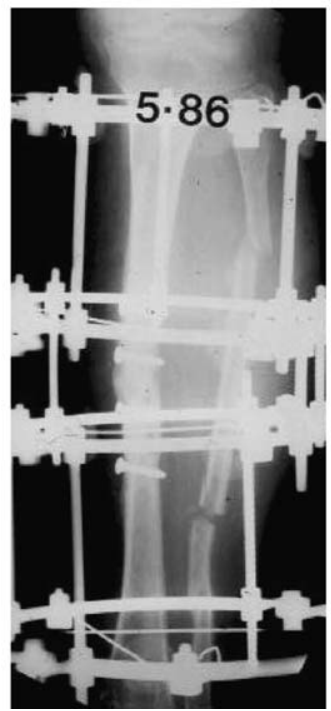

(d)

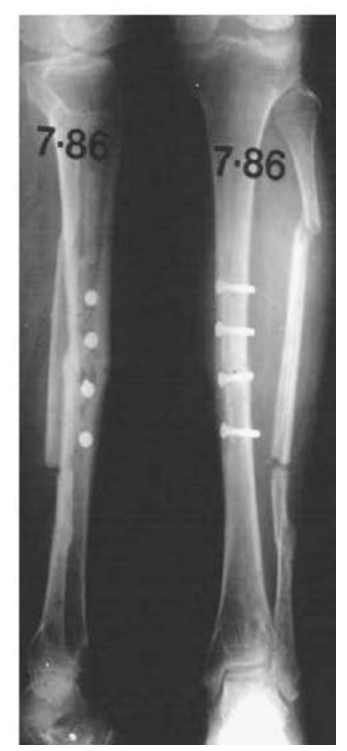

Figure 1 (a) Type 1 non-union. (b) X-ray of type 1 non-union: morphology and not presence of bone is discriminant for classification (case 1). (c) Treatment with external fixation of type 1 non-union: mechanical close treatment and not bone grafting, is discriminant for the treatment (case 1). (d) End point treatment: healing of type 1 non-union after 3 months (removal of frame).

effect on the consolidation which, at times could also involve axial alterations. Stabilization of this kind of morphology requires a complex and difficult inter-fragmented compression (Fig. 2).

Type 3 non-unions have serious comminution or atrophy of the stumps which often result from exposed fractures with bone loss or post-infection consequences. At times this type of fracture seems to have a simple morphology, but part of the bone that completes the rim, which seems to be uncomplicated, is indeed necrotic. A typical case is a nonunion after plate synthesis with cortical necrosis underneath the plate itself. The radiographic aspect of this kind of bone sclerosis has a completely different significance to the thickening of the edges in the kind of non-union described by Judet et al. ${ }^{3}$ In this case, a purely mechanical stabilization is not sufficient or simply impossible to perform.

Type 4 non-unions are infected fractures, whatever the radiographic aspect. The removal of the infected area is the dominating choice of treatment.

It goes without saying that non-unions types 1 and 2 require a purely mechanical treatment, whilst types 3 and 4 require a biological one. Mechanical non-unions are superimposible to those described by Weber and $\mathrm{Cech}^{10}$ as vital, whilst type 3 are superimposible to those defined as being not vital.

In our institution, the external fixator is the instrument of choice to achieve the principle of mechanical stabilisation for both vital and mechanical non-unions.

\section{Rational for the use of external fixation in mechanical non-unions}

A non-union site is always an area with a risk of biological compromise. This risk involves poor vascularization both on the periostial and endosteal side, if the non-union site is opened and a plate or nail osteosynthesis performed, then the situation is further compromised with an increased risk of infection.

In order to obtain the maximum benefit from the mechanical stimulus, the load must be varied throughout the treatment phase. Many authors have demonstrated that although compression is necessary during the early stages of fracture healing ${ }^{5,8,11}$ loading must be cyclic at a later stage, a principle applied not only to fractures but also to non-unions, as demonstrated by llizarov. ${ }^{4}$ External fixation not only responds to the first requirements but it is the only aid which allows a mechanical modulation of the healing process. In the simple less complicated type 1 non-unions, if there is no relevant axial deformity, a less cumbersome simpler fixator may be used, such as the monolateral type. The only requisite is that there must be the possibility to convert the rigid module into a dynamic one at such 


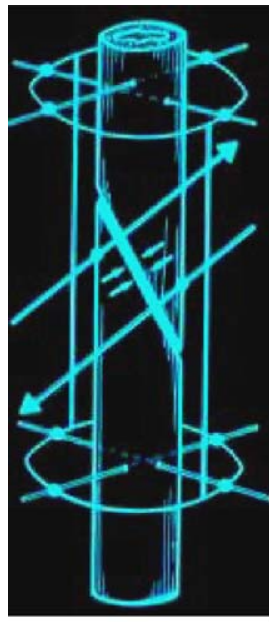

(a)

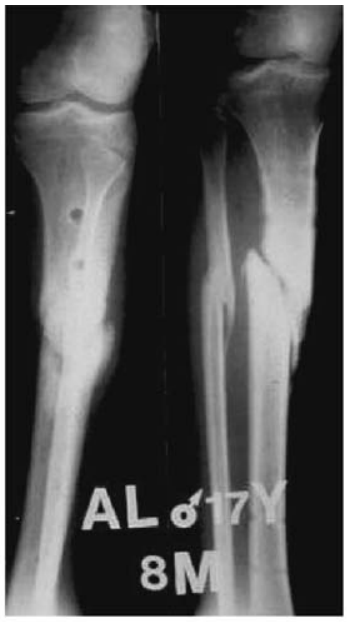

(b)

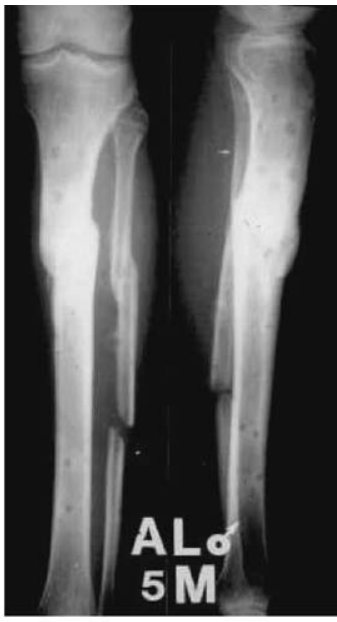

(d)

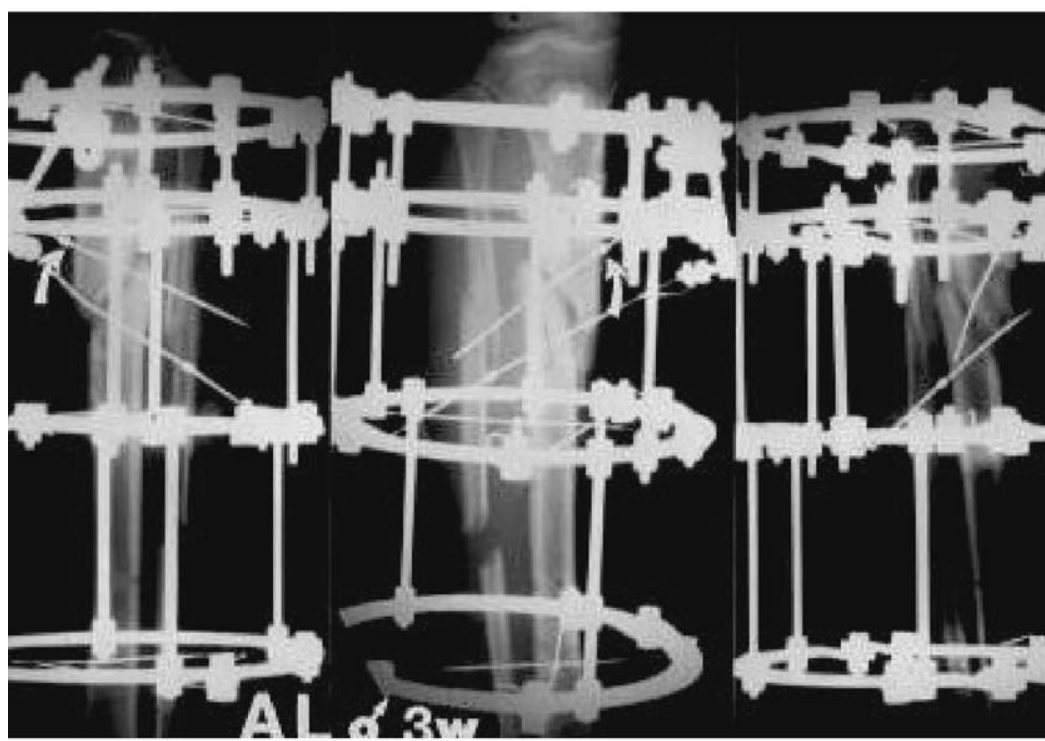

(c)

Figure 2 (a) Type 2 non-union. (b) X-ray of type 2 non-union. (c) Treatment with external fixation of type 2 non-union. (d) End point treatment: healing of type 2 non-union after 5 months.

times as the healing process requires it. The Orthofix fixator meets these requirements well. ${ }^{6}$

The external fixation of a type 2 non-union (under closed surgery) is a more complex situation in as much as the morphology itself is more complex. Here, a monolateral fixator does not fulfil the stability requirements in the early stages as it applies only negative cutting and torsional forces. The most appropriate fixator is the circular one such as the Ilizarov. Thanks to the crossed wire assembly, this fixator not only allows for a fragmented compression on an oblique rim site, but also converts the otherwise negative torsional and cutting forces into axial force. $^{1}$

There is also another advantage in using the circular fixator being that the load force created is absorbed by the bone automatically as the con- solidation and rigidity of the bone increases, thanks to the flexibility of the wires. This is the very concept of the automatic dynamic action of the Ilizarov. This takes place as long as the elements that fix the bone are the thin elastic wires used in the original model. Indeed, the ever more common use of circular fixation with larger screw diametres (from 4.5 to $6 \mathrm{~mm}$ ) rather than the thin original wires, limit this function, and should, therefore, be kept to a minimum indispensable in the case of non-unions.

\section{Rational for the use of external fixation in type 3 non-unions}

It is not possible to adopt a mechanical treatment for type 3 non-unions. The advent of the Ilizarov 
principle of distractional osteogenesis has revolutionized the treatment of this type of non-union. There are two possible techniques: a monofocal compression or a bifocal distraction on the nonunion site with compression on the pseudoarthrosis site. The first technique is to be applied in the presence of a bone loss of less than $3 \mathrm{~cm}$ or in type 1 non-unions with shortening of the limb. The site is first compressed and then distracted; the new bone formation leads not only to the healing of the nonunion, but also corrects the hypometry.

The second technique is used in the presence of a more extensive bone loss; apart from bone compacting, the non-union site must be resected as it is better to substitute a poorly biological atrophic bone area with two bone surfaces of good quality modelled in such a way as to allow for an easy stabilization under compression. The newly formed bone at the site of compacting is able to fill in all the gaps that have been created. The stability of the fixation allows for immediate functional recovery, there is practically no risk of infection, the new bone formation is completely normal and does not require the long integration times needed in the case of a transplant.

\section{Indications for the use of traditional bone grafting}

Traditional bone grafting such as autologous iliac crest cancellous graft may be indicated for us in type 3 non-unions in the presence of necrotic areas that do not involve the bone, without serious atrophy. Here, the use of a simple compression may not be able to achieve healing of all the necrotic area, whilst the resection of the non-union site may be "overcare" involving the sacrifice of a large quantity of vital bone. The most productive technique is that of taking a spongy cortical graft from the iliac wing and to press fit it into the non-union site, in the point of necrotic bone removal. An external fixation under compression allows for mechanical stabilization, which has been demonstrated to favour the integration of the transplant in the early stages. ${ }^{9}$

Traditional bone grafts (allogenic, synthetic) may also be indicated in non-unions treated with the bifocal technique, in as much as, sometimes, the transplant is necessary for the healing of the contact point of the re-sectioned stumps.

\section{Rational in the use of external fixation in type 4 non-unions (septic)}

In this case, re-sectioning as aforementioned allows for the excision of the infected tissue. The septic non-union is, in fact, transformed into an aseptic one of type 3. As there is no problem of quantity of bone loss, the cleansing may be radical. Also, the soft infected tissues are removed and the wound left open thus creating a true Papineau timing. The soft tissues are closed when they come into contact with the bone stumps, this procedure sometimes requires some minor plastic surgery.

\section{Rational in the use of bone morphogenetic protein (BMP7) in the biological treatment of non-unions}

The clinical introduction of BMP-7 (OP1) provides the possibility to stimulate osteoprogenitor cells to regenerate bone. The gold standard in OP1 use is the work of Friedlander et al. ${ }^{2}$ In our treatment protocol OP1 is not used in bone losses as in this case we apply Ilizarov technique. We use OP1 to stimulate bone consolidation when biological power is limited or autologous or allogenic bone transplantation is not possible or safe in situations where we have:

- Traditional technique failure.

- Traditional technique being unfeasible.

- Delayed union of docking point, when using Ilizarov technique.

\section{Patients and methods}

A total of 287 long bone non-unions were treated in 279 patients from 1982 to 2004 . Average age was 32 years $(8-80)$ with the highest frequency being between 19 and 38 years of age. There were 172 tibia, 86 femurs and 29 bones from the upper limbs.

The average time lapse from injury to treatment protocol was 11.8 months (2-92) although it was not possible to obtain a precise clinical history for 12 patients.

There were 158 type 1 non-unions, 43 type 2, 17 type 3 and 69 infected ones (type 4). An average of $4.7 \mathrm{~cm} \mathrm{(2-10)} \mathrm{bone} \mathrm{loss} \mathrm{was} \mathrm{observed} \mathrm{in} 59$ cases, 34 of which were type 4 non-unions, 11 were type 3 and 10 type 2. Bifocal compression/distraction was used in all cases but two, where a monofocal compression/distraction was used to recover gaps of about $3 \mathrm{~cm}$. No major plastic surgery was required for any of the cases.

A total of 101 Orthofix devices were used, 5 Hoffmann, 1 Exfire and 197 Ilizarov in the cases that achieved healing with only one device. The type of fixator was alternated according to the individual requirements in 28 cases.

The mechanical properties used in 99 cases were that of alternated compression/distraction, pure 
compression in 117, bifocal compression/distraction in 57 and distraction in six with neutralization in eight.

Autologus bone grafting was used in 35 cases $(11 \%)$, twice to complete the healing of the contact points after bifocal technique and six times directly on the pseudarthrosis site.

Four fractures, or rather mobilization of an insufficiently consolidated non-union site was observed after removal of the external devise in seven cases. The devices were replaced and healing was achieved in all cases.

\section{Results}

A total of 266 consolidations were achieved in 287 segments (93\%). "Healing" was considered such a time when the segment treated had complete functional recovery, or in the case of infection when there was a complete resolution. The period in which crutches were used after removal of the devices, is included in the healing time.

From 30 September 2002 to 01 August 2005, 31 patients, mean age 38 years (range: 22-65), (32 cases, 33 doses of OP1) have been treated with OP1. Before last intervention, mean number of operations was five (range: 3-26) with mean time of treatment 3 years (range: 1-31). Fifteen tibias, 11 femurs, 3 humerus and 3 forearms were treated. Fracture healing was successful in 20 cases, seven are still under treatment and two have been declared as failures (one osteomyelitis relapse). The mean time of union was 4 months (range: 26). Fracture healing was evaluated by both clinical and radiological assessment (presence of callous bridging two cortices on two X-ray planes). Radiological bone consolidation did not correlate with clinical stability and functional recovery.

\section{Discussion}

We are of the opinion that a $93 \%$ healing for this type of pathology seems to be a good result especially in the light of the fact that more than $30 \%$ of the cases were complex and $25 \%$ had infections. Moreover, most importantly all the infections were treated successfully. This can be attributed to the fact that out treatment strategy allows for a radical debridement of the infected site without fear of creating a disabling bone gap and without cumbering the bone with an osteosynthesis device. Moreover, there was a drastic reduction in the use of bone grafting procedures, reducing a recognised risk of triggering a re-infection.
Any axial deviations, hypermetries and rigidities were limited to the most complex cases in which the functional recovery of the limb may be considered a success in itself. As to the 18 cases where there was a residual rigidity of the knee, it must be noted that nine were pre-existent and nine were posttreatment. Three of these were infected non-unions (one was a type 3 ).

Types 1 and 2 cases represented $70 \%$ of the nonunions. Pure compression was applied in 99 cases versus 117 of alternated compression/distraction. This would make one think that there was a purely mechanical treatment in $34 \%$ and some form of biological stimulation in $42 \%$. In these cases one is not dealing with a loss of bone substance, which benefit from techniques such as both mono and bifocal compression/distraction. In truth, it is difficult to say whether there has been a purely biological or mechanical treatment. What one can say, however, is that anyway stabilization has been achieved and that the biological stimulation is an intrinsic element to the fixator for the very characteristics of the llizarov.

When dealing with the issues related to the use of the fixator such as the breaking of wires or inflammatory reactions (superficial infections), it is best to clear up once and for all that these do not in fact represent "complications" as such but rather intrinsic factors in the methodology itself. What should be evaluated, however, is if one is dealing with elements that may lead to a refusal of the use of this method. When examining the cost/benefit ratio and the results obtained the obvious answer is no. Moreover, the correction of these conditions does not require major surgery, as at most it entails substitution or limited surgical cleansing. The substitution of the devices during the course of the treatment period does not represent a drawback but rather an advantage. Indeed, the mechanical and biological requirements may vary in the most complex cases depending on the treatment stage.

One such example is that of major bone loss, firstly the gap must be filled and then what has been obtained brought to healing. It is then possible to pass from a cumbersome fixator like the Illizarov to a simpler less cumbersome one, like a monolateral device or a Hoffmann. The stabilisation elements already in place may be utilized, or substituted party or in the whole, depending on the conditions of the device, with minor surgery. This option is particularly helpful when dealing with the femur or humerus, an area in which devices are badly tolerated.

Re-fracturing may occur after the removal of the fixator. All of which is tied, in part, to the condition 
of the bone and in part to the evaluation difficulty of the mechanical hold of the consolidation achieved. Of the four cases reported as "fractures", only one was a "real fatigue fracture" of an insufficient bone bridge, whilst the other three were a too early mobilization due to insufficient hold. Whilst the first issue has its aetiology in a technical defect (the bone stumps are modelled adequately during re-sectioning and the contact point must be obtained without movement), the second one is more difficult to identify. Surely the instrumental evaluation techniques as to rigidity and consolidation should be improved and made more readily available.

The total average healing time of 7 months, although in line with that reported in literature on the same pathology, should be evaluated in an analytical manner on the basis of the grade of the pathology involved. When the healing times are analysed as to type, there is no significant difference per segment or per type. That means that the femur type 1 non-union, heals more slowly than does the tibia type 1 and those of type 3 heal more slowly than the tibia type 3 . These differences, are not, however, significant as is true for the difference in the healing times among types 1-4 non-unions. These values lead us to some conclusions that types 1 and 2 non-unions, heal more quickly than those that require a biological treatment or a bone graft. It is therefore true that tissue can transform itself into bone, thanks to a correct mechanical stimulation. Also, types 3 and 4 non-unions do heal, but they require longer times and direct intervention. When types 3 (aseptic) and 4 (septic) are taken into consideration, they heal in much the same way, except that the septic non-union heals a little quicker without a significant difference.
OP-1 administration provides the possibility to increase biologic stimulation with minor risks and more efficacy, but its significance is not dissimilar to our reported rational of treatment.

\section{References}

1. Cattaneo R, Villa A, Catagni M, Tentori L. Treatment of septic or non-septic diaphyseal pseudoarthroses by llizarov's monofocal compression method. Rev Chir Orthop Reparatrice Appar Mot 1985;71(4):223-9.

2. Friedlaender GE, Perry CR, Cole JD, et al. Osteogenic protein-1 (bone morphogenetic protein-7) in the treatment of tibial nonunions. J Bone Joint Surg Am 2001;83(Suppl. 1): S151-8.

3. Judet R, Judet J, Roy-Camille R. La vascularisation des psedarthroses des os longs d'après una ètude clinique et expèrimentale. Rev Chir Orthop 1958;44:381-5.

4. Ilizarov GA. The tension-stress effect on the genesis and growth of tissues. Part II. The influence of the rate and frequency of distraction. Clin Orthop Relat Res 1989;(239): 263-85.

5. Kenwright J, Richardson JB, Cunningham JL, et al. Axial movement and tibial fractures. A controlled randomised trial of treatment. J Bone Joint Surg Br 1991;73(4):654-9.

6. Marsh JL, Nepola JV, Meffert R. Dynamic external fixation for stabilisation of nonunions. Clin Orthop Rel Res 1992;278:200-6.

7. Muller ME. Treatment of nonunion by compression. Clin Orthop 1965;43:83-92.

8. Panjabi MM, White III AA, Wolf Jr JW. A biomechanical comparison of the effects of constant and cyclic compression on fracture healing in rabbit long bones. Acta Orthop Scand 1979;50(6 Pt 1):653-61.

9. Stevenson S. Enhancement of fracture healing with autogenous and allogeneic bone grafts. Clin Orthop Rel Res 1998;355(Suppl.):S239-46.

10. Weber BG, Cech O. Pseudarthrosis. Bern: Hans Huber Publishers, 1976.

11. Wolf Jr JW, White III AA, Panjabi MM, Southwick WO. Comparison of cyclic loading versus constant compression in the treatment of long-bone fractures in rabbits. Bone Joint Surg Am 1981;63(5):805-10. 